Relato de Caso / Case Report

\title{
Tratamento de hemangioma gigante com interferon alfa: relato de dois casos
}

\section{Treatment of giant hemangioma with interferon-alpha: report of two cases}

Ana Julia Balau ${ }^{1}$

Livia C. De Nadai ${ }^{1}$

Mônica S. Bressan ${ }^{2}$

Josiane L. Simão ${ }^{1}$
O objetivo do trabalho é descrever o uso de interferon alfa no tratamento de pacientes com hemangioma gigante. Os autores relatam e analisam dois casos de hemangioma gigante em tratamento com interferon alfa. IBS, 3 anos, em acompanhamento no Ambulatório de Hematologia desde um ano de idade com quadro de lesão angiomatosa em praticamente toda hemiface direita, acompanhada de sangramentos gengivais importantes. Após a realização de exames complementares (Angiorressonância magnética) e feito o diagnóstico de hemangioma gigante em face, foi iniciado tratamento com prednisona e, posteriormente, associação com interferon alfa e observada importante melhora do quadro, resultando na diminuição dos episódios de sangramento e no tamanho do tumor. C.N.P., 12 anos, apresentando nódulo em região lateral de joelho esquerdo há 2 anos, com aumento progressivo do tamanho e dor local. Fez uso de prednisona e, sem melhora do quadro, introduzido interferon alfa com regressão importante do tamanho do tumor. O tratamento com interferon alfa deve ser considerado no tratamento de hemangiomas, pois apresenta bons resultados em relação à diminuição do tamanho do tumor e, conseqüentemente, reduz as intercorrências clínicas associadas à sua presença, principalmente os sangramentos. Rev. bras. hematol. hemoter. 2007;29(4):406-411.

Palavras-chave: Hemangioma; interferon alfa; sangramento; criança.

\section{Introdução}

Hemangiomas são os tumores mais comuns da infância, com incidência em recém-nascidos de 1\%-3\% e alcançando índice de $10 \%-12 \%$ nas crianças até 1 ano de idade. ${ }^{1,2}$ De origem vascular, são decorrentes da rápida proliferação de células endoteliais, e, segundo Gontijo, ${ }^{2}$ apresentam fases consecutivas de crescimento, estabilização e regressão. Podem estar ausentes ao nascimento ou apresentarem-se como marcas cutâneas, que possuem capacidade de crescimento rápido, seguido de um período de regressão, até a involução completa em grande parte dos casos.

Predominam no sexo feminino, em crianças de pele clara e há uma maior incidência em prematuros, especialmente naqueles de peso menor que $1.500 \mathrm{~g}$ ao nascimento. ${ }^{2}$ Segundo Finn et al, ${ }^{1}$ aproximadamente $80 \%$ dos pacientes apresentam lesões únicas, acometendo preferencialmente a pele e estão distribuídos mais freqüentemente no tronco (25\%) e cabeça e pescoço (60\%). ${ }^{1}$ Mulliken e Glowacki propuseram uma nova classificação para as lesões vasculares diferente daquela proposta por Virchow e Wegener em 1877, dividindo as lesões em hemangiomas e malformações vasculares. ${ }^{3}$ Atualmente são divididos em tumores e malformações vasculares, classificação adotada oficialmente pela Sociedade Internacional para Estudo das Anomalias Vasculares. Com base nesta última classificação, tumores vasculares incluem hemangiomas da infância, hemangioma congênito rapidamente involutivo, hemangioma congênito não involutivo, angioma em tufos, hemangioendotelioma kaposiforme e granuloma piogênico, enquanto malformações vasculares são erros de morfogênese classificados de acordo com o vaso predominante. ${ }^{2}$

As complicações mais freqüentes dos hemangiomas são de origem estética ou funcional, a depender de seu tama-

\footnotetext{
${ }^{1}$ Aluna da graduação do sexto ano de Medicina (2006), Faculdade de Medicina de Marília (Famema), Marília, SP.

${ }^{2}$ Médica assistente de ensino do Ambulatório de Hematologia Pediátrica da Faculdade de Medicina de Marília.
}

Correspondência: Livia Chiosini De Nadai

Rua Marquês de Itu, 545, ap.42 - São Paulo-SP - Brasil

Tel.: (11) 3554-7616 / (11) 8308-1836

E-mail: liviadenadai@hotmail.com 
nho e localização. De acordo com Chiller et al, ${ }^{4}$ podem ocorrer ulcerações em $21 \%$ dos casos e sangramentos em 7,5\%. Além disso, podem causar obstruções de vias aéreas e dificuldades de deglutição, em caso de hemangiomas cervicais e faciais extensos, insuficiência cardíaca, hipotireiodismo e alterações visuais, como astigmatismo, proptose, ambliopia. ${ }^{5}$

Como grande parte dos hemangiomas são assintomáticos e ainda podem regredir espontaneamente, nem sempre existe a necessidade de tratamento. Entretanto, 5\% a $10 \%$ das crianças com este diagnóstico desenvolvem deformidades funcionais e outras alterações que ameaçam a vida, justificando a intervenção terapêutica. ${ }^{3}$ Tratamentos com corticoesteróides, embolização arterial, cirurgia, crioterapia, radioterapia, quimioterapia, laser e interferon alfa têm sido usados freqüentemente, sendo o uso de corticoesteróides a opção mais escolhida até o momento. ${ }^{6}$

Os autores relatam os casos de duas crianças com hemangioma tratadas com sucesso com o uso do interferon alfa.

\section{Relato de Casos}

\section{Caso 1}

I.B.S., sexo feminino, três anos de idade, em acompanhamento no Ambulatório de Hematologia da Faculdade de Medicina de Marília desde um ano de idade, com diagnóstico de hemangioma gigante em face.

Na sua primeira consulta (28/10/2003), a mãe relatava que a paciente havia apresentado sangramento gengival importante devido à erupção dentária em arcada dentária superior direita, tendo evoluído para choque hipovolêmico com hemoglobina de 3,9g/dL. Apresentava-se ainda descorada e trazia exames realizados na cidade de origem: angiorressonância magnética com laudo sugestivo de malformação vascular em face à direita, evidenciando borramento no tecido subtentorial em região anterior direita da face por presença de inúmeras imagens tubuliformes, que representavam vasos, e se extendia desde a órbita até o ângulo da mandíbula.

Ao exame notava-se lesão hemangiomatosa atingindo toda hemiface direita, principalmente em região infra-orbitária, com infiltração em palato e sangramento ativo no local da erupção dentária (molares superiores à direita). Havia acometimento de mucosa nasal e toda mucosa jugal, ambas à direita.

Na ocasião, a paciente estava em uso de prednisona $4 \mathrm{mg} / \mathrm{kg} /$ dia há sessenta dias, com discreta redução do tumor, estimada em aproximadamente $10 \%$. Foi reduzida a dose da prednisona para $2 \mathrm{mg} / \mathrm{kg} /$ dia e mantida por mais sessenta dias. Sem redução expressiva no tamanho do tumor, optouse pela introdução de interferon alfa na dose de 100.000 UI/ kg três vezes por semana e pela manutenção da prednisona $2 \mathrm{mg} / \mathrm{kg} / \mathrm{dia}$ por mais quatro semanas. Após este período, reduzimos a dose de prednisona para $1 \mathrm{mg} / \mathrm{kg} /$ dia e mantivemos a mesma dose de interferon, porém em duas aplicações

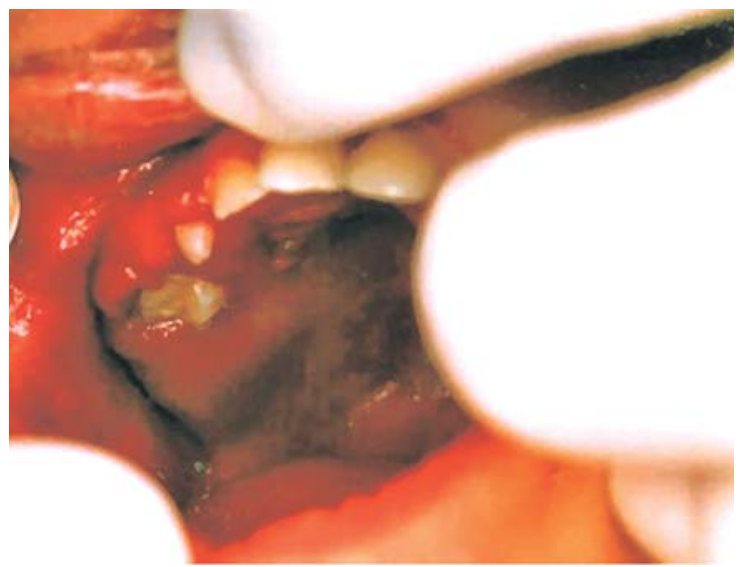

Figura 1. Hemangioma em face, acometendo região jugal, antes de iniciado o tratamento

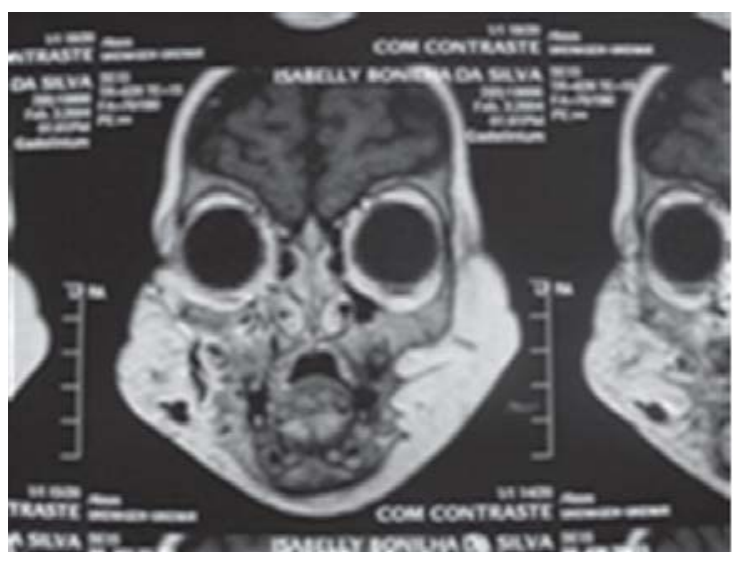

Figura 2. Ressonância nuclear magnética mostrando acometimento do tumor

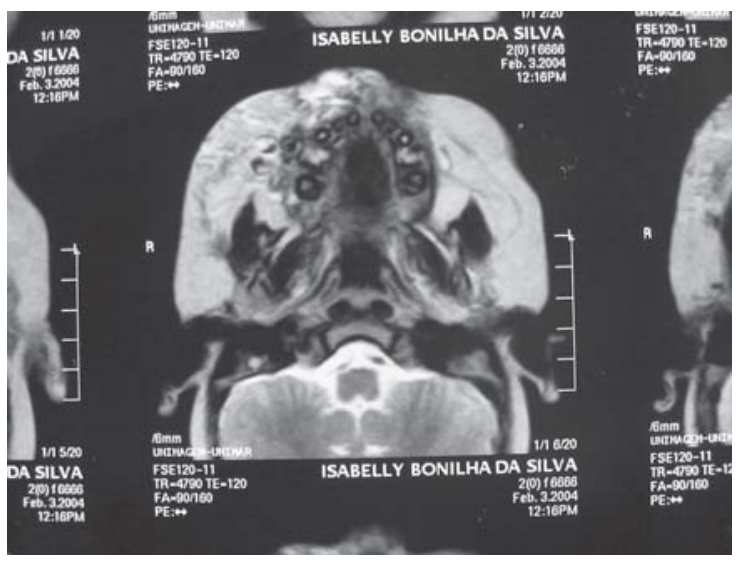

Figura 3. Ressonância nuclear magnética mostrando acometimento do tumor

semanais.

O tratamento seguiu-se desta forma por mais 24 semanas, sendo que no primeiro ano foi observada melhora importante do quadro, principalmente quanto à infiltração da lesão em arcada dentária, com redução estimada de 30\% em 


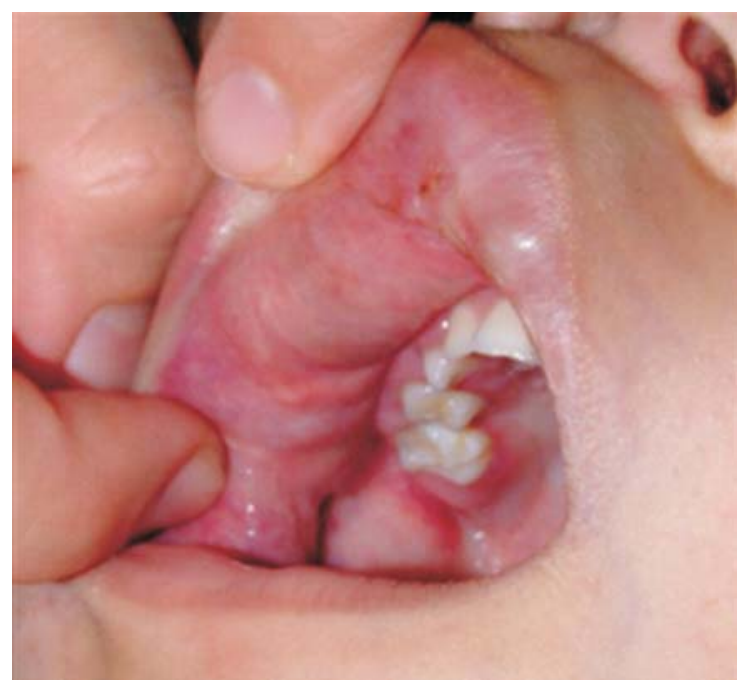

Figura 4. Redução do tumor em região jugal após tratamento com interferon alfa.

seu tamanho. Além disso, nesse mesmo período houve apenas dois episódios de sangramento de leve a moderada intensidade, sendo um deles por erupção dentária e outro, epistaxe espontânea. Nas consultas posteriores houve retirada gradual da prednisona até sua interrupção e aumento da dose de interferon para $150.000 \mathrm{UI} / \mathrm{kg}$ em duas aplicações semanais.

Após 23 meses de tratamento com interferon, a paciente foi submetida a nove sessões de esclerose vascular com melhora discreta e temporária. Em atendimento recente, a paciente apresentava-se bem, sem sinais de sangramento e sem aumento do tamanho tumoral até a última consulta, quando perdemos o contato uma vez que a paciente mudou-se de cidade.

\section{Caso 2}

C.N.P., 12 anos, sexo feminino, procedente de Canitar.

Paciente veio encaminhada da cidade de origem com quadro de hemangioma em membro inferior esquerdo há 8 anos, já tendo feito duas ressecções com sucesso há 4 e 3 anos. Há 2 anos, notou nódulo em região lateral de joelho esquerdo, associado a dor local e aumento progressivo de tamanho, sem febre ou alterações de movimentação. Fez uso de prednisona $1 \mathrm{mg} / \mathrm{kg} / \mathrm{dia}$ por quatro semanas sem melhora.

Ao exame apresentava-se descorada $+/ 4+$, hidratada, anictérica, acianótica e afebril, com fácies cushingóide.

Joelho esquerdo com presença de pequeno abaulamento em região lateral, móvel e indolor. Circunferência de joelho esquerdo medindo $32 \mathrm{~cm}$, direito de $31 \mathrm{~cm}$; panturrilha direita com $28 \mathrm{~cm}$, esquerda com $30 \mathrm{~cm}$; tornozelo direito com $19 \mathrm{~cm}$ e esquerdo com $21 \mathrm{~cm}$. Sem outras alterações no exame físico. Exames laboratoriais: $\mathrm{Hb}=13,8 \mathrm{~g} / \mathrm{dL} ; \mathrm{Ht}=41,4 \%$; Leucócitos $=9.300$, plaquetas $=276.000$.

Trouxe apenas laudo de angiografia por ressonância nuclear magnética realizada em outro serviço, que revelou

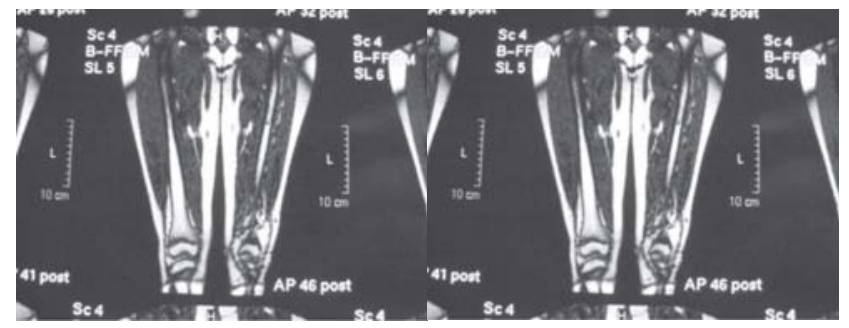

Figura 5
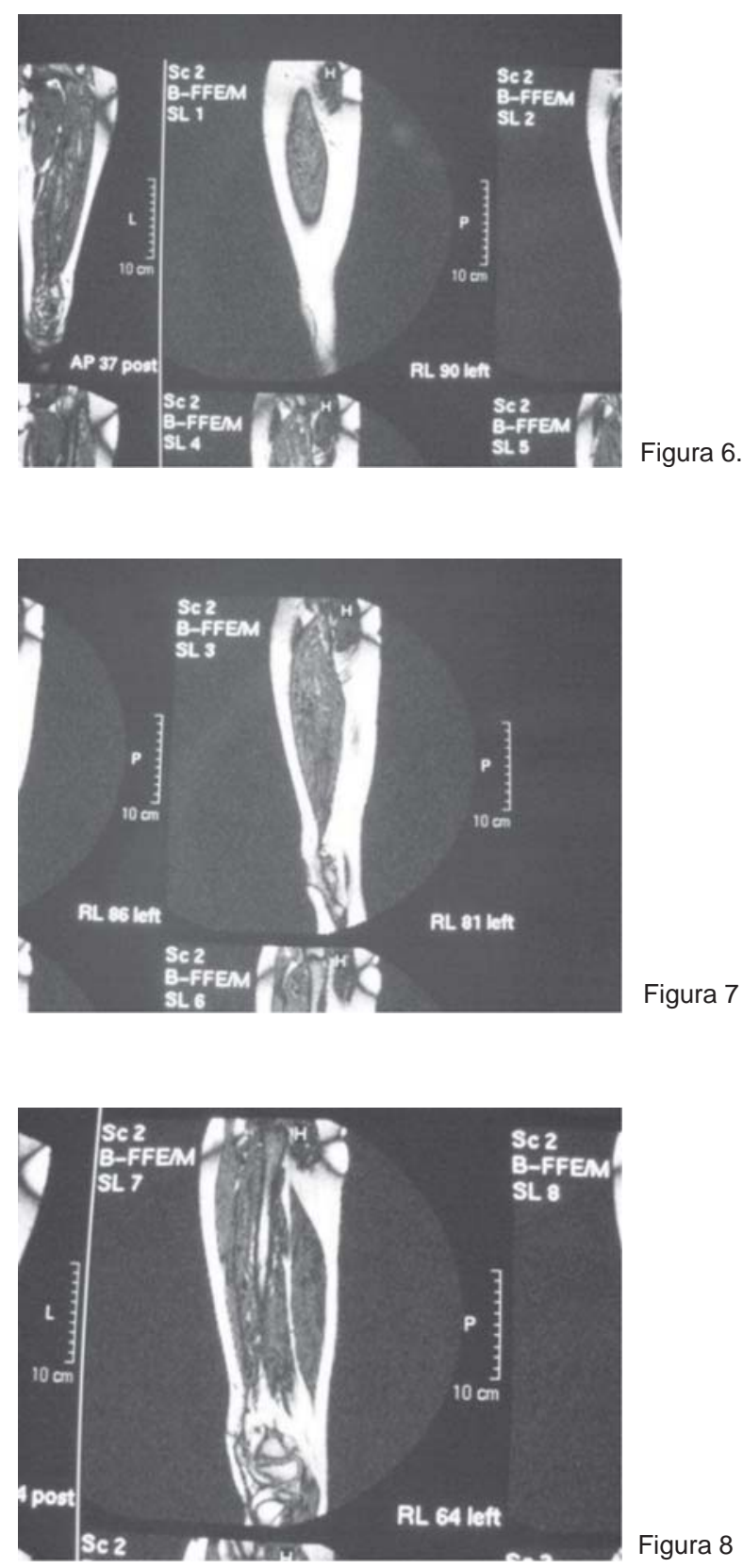

Figuras 5-8. Angiografia por ressonância nuclear magnética evidenciando hemangioma venoso em face lateral de coxa esquerda, terço distal após uso de interferon alfa, medindo $2,8 \times 1,9 \mathrm{~mm}$. 
artérias femorais, poplíteas e troncos fibulares de origem, calibre e contornos normais. Presença de formação de lesão expansiva amorfa, de característica vascular, medindo cerca de 45x30mm, localizada ao nível de fêmur distal, em seu aspecto lateral.

Introduzido interferon 3000000 UI duas vezes por semana. Um mês após o início do tratamento, notou-se uma redução expressiva do tamanho do tumor, de 45x30mm para 28x19mm, conforme dados de angiografia por ressonância magnética de membro inferior esquerdo.

\section{Discussão}

Segundo Mulliken e Glowacki, hemangiomas são tumores vasculares que apresentam duas fases, uma fase proliferativa de crescimento rápido e hipercelularidade, com início imediatamente após o nascimento e com duração até os próximos 9 a 12 meses, seguida de uma fase involutiva, com predomínio da apoptose sobre a mitose, com duração de 5 a 7 anos. ${ }^{2,3,7}$ São compostos por endotélio vascular proliferante, fibroblastos e mastócitos que crescem, estabilizam e então regridem em um processo de fibrose e diminuição da celularidade. $^{6}$

Caracterizados por crescimento pós-natal rápido, que dura até os 6 a 10 meses de idade, raramente excedendo os 2 anos, é seguido por uma fase quiescente que persiste por alguns meses e por uma fase de involução lenta. Metade das lesões regride até os 5 anos de idade, $70 \%$ até os 7 anos e $90 \%$ até os 10 anos, o que justifica a conduta expectante na maior parte dos casos. ${ }^{2,6} \mathrm{~A}$ involução ocorre em lesões nas quais há proliferação das células do endotélio vascular. As lesões em que esta proliferação não ocorre são consideradas como malformações vasculares, apresentam ciclo celular normal e, portanto, não involuem. ${ }^{8}$

Apesar de pouco esclarecida a sua fisiopatologia, considera-se que seja resultado de um desequilíbrio na angiogênese, que permite a proliferação descontrolada de elementos vasculares. ${ }^{2}$

Mais da metade dos hemangiomas distribui-se na região de cabeça e pescoço, em alguns locais específicos, o que provavelmente está relacionado com as linhas de fusão embrionárias e com o desenvolvimento dos metâmeros faciais, o que também explica a maior parte de suas complicações. ${ }^{3}$ Porém, são também freqüentes no fígado e Sistema Nervoso Central.

No fígado, é o tumor mais freqüente no primeiro ano de vida e é raro após os 3 anos de idade. Caracteriza-se pelo crescimento rápido nos primeiros meses e regressão completa por volta dos 5 a 7 anos em $75 \%$ dos casos. Apresenta-se com hepatomegalia e, apesar de benigno, pode atingir grandes dimensões e levar a complicações, como insuficiência cardíaca por formação de shunts arteriovenosos em 50\% dos casos, levando a óbito em $70 \%$ desses pacientes, caso não tratados adequadamente. ${ }^{9}$
São clinicamente heterogêneos, sendo que sua apresentação varia de acordo com o tamanho e local acometidos, profundidade da lesão e estágio de evolução. ${ }^{2}$ No recémnascido, podem originar-se como uma lesão macular hipocrômica associada a telangiectasias e, com a proliferação do tumor, assume suas formas mais conhecidas: placa levemente elevada, de coloração vermelho-vivo, que não desaparece à vitropressão. ${ }^{10}$

Outra maneira de se classificar os hemangiomas é de acordo com sua profundidade: superficiais, profundos ou combinados. Os superficiais representam 50\%-60\% dos casos, com limites bem definidos, podendo ser nodulares ou em placa, cor vermelho-vivo, com pele normal adjacente, restritos à derme papilar e reticular. Já os profundos (15\%), ou cavernosos como na antiga classificação, são nodulares, de tom da pele ou azulado, podendo haver telangiectasias superficialmente, acometendo a derme profunda e o tecido subcutâneo. ${ }^{2}$

Haggstrom et a ${ }^{11}$ observaram 1.058 pacientes durante 13 meses (setembro 2002- outubro 2003) que apresentavam menos de 12 anos e mais de um hemangioma em qualquer estágio de evolução, totalizando 1.915 hemangiomas. Neste estudo, observou-se que 68,8\% dos pacientes apresentavam uma única lesão e a maioria tinha até seis hemangiomas (97\%). Quanto ao local mais freqüentemente acometido, 41\% localizavam-se na face, $23,3 \%$ no tronco, $21 \%$ na região de cabeça e pescoço, excluindo-se a região facial, $18,4 \%$ nas extremidades e $6,1 \%$ no períneo; $66,8 \%$ foram classificados como localizados, $16,5 \%$ como indeterminados, $13,1 \%$ segmentares e 16,5 como multifocais. Vinte e quatro por cento desses pacientes apresentaram algum tipo de complicação que estava relacionada ao tamanho (média de $37,3 \mathrm{~cm}^{2}$ ), localização (mais comuns no períneo e face) e subtipo (segmentares e indeterminados) e $38 \%$ receberam algum tipo de tratamento (12,3\% receberam corticoesteróides sistêmicos; 4,1\% corticoesteróides intralesionais e 9,8\% na forma tópica; 8,0\% receberam pulsoterapia com laser e $5,7 \%$ passaram por cirurgia excisional).

O diagnóstico de hemangioma é feito com base na história e achados clínicos e devem ser diferenciados das malformações vasculares e de outros tipos de tumor. Para isso, uma avaliação radiológica se faz útil e pode ser realizada através de ultra-sonografia com doppler e tomografia computadorizada, sendo o padrão-ouro a ressonância magnética, melhor exame para confirmar as características teciduais da lesão, sua extensão e avaliar anomalias adjacentes associadas. ${ }^{2,11}$

Alguns autores estimam que apenas 10\%-20\% dos hemangiomas precisam ser tratados, sendo estes os que apresentam complicações, como o comprometimento ocular, obstrução de vias aéreas, hemorragias, ulcerações e complicações estéticas. Devem ser considerados a idade do paciente, tamanho, número e localização das lesões, seu estágio evolutivo e sintomas associados. ${ }^{2}$ 
Diversos tipos de tratamento têm sido utilizados, como a embolização arterial, corticoterapia, cirurgia, radioterapia, quimioterapia, laser e interferon alfa, sendo a corticoterapia o tratamento de primeira escolha. ${ }^{6}$

Numa tentativa de diminuir as complicações e minimizar os efeitos colaterais dos corticoesteróides, vem sendo cada vez mais utilizada a terapia com interferon alfa.

O mecanismo de ação do interferon alfa não é totalmente conhecido, mas sabe-se que ele inibe a migração e proliferação das células endoteliais in vitro e a angiogênese in vivo, exercendo um efeito supressor sobre o endotélio vascular, inibindo a proliferação de células endoteliais e a produção de substâncias estimulantes da angiogênese, como o fator de crescimento fibroblástico beta (bFGF). ${ }^{6}$

Diversos trabalhos têm comprovado a eficácia do interferon no tratamento dos hemangiomas. De acordo com diferentes autores, o uso de interferon alfa consegue uma redução de mais de $50 \%$ do tamanho em aproximadamente $58 \%$ dos casos e, em $26 \%$, uma redução de $20 \%-40 \%$. $^{12}$ Chang et al analisaram dez estudos abrangendo 73 pacientes, num período médio de tratamento de dez meses, onde foi observada, em $63 \%$ dos casos, uma melhora de mais de $50 \%$ das lesões ao exame físico, bem como nos exames de imagem. ${ }^{13}$

Portanto, tanto o interferon alfa 2a como $2 \mathrm{~b}$ têm sido empregados nos hemangiomas resistentes ao tratamento com corticoesteróides. ${ }^{10}$ Greinwald et $a l^{12}$ recomendam aumento gradual da dose de 1 milhão de unidades internacionais a cada mês, até atingir 3 milhões UI/ $\mathrm{m}^{2} /$ dia, por via subcutânea, durante dois a seis meses. Segundo Hagberg et $a l^{14} \mathrm{e}$ Takahashi et al, ${ }^{15}$ o tratamento deve ser mantido por mais de um ano, uma vez que a melhora dos sintomas foi observada após 10 a 19 meses após o início da terapia com interferon alfa.

Apesar de bem tolerado pela maior parte dos pacientes, efeitos adversos são freqüentes, como febre durante os primeiros dias de administração, hiporexia, aumento discreto de enzimas hepáticas, neutropenia transitória e sintomas neurológicos, que podem ocorrer em até $25 \%$ dos casos e são aparentemente dependentes da dose. Além disso, ainda podem ocorrer enfraquecimento dos cabelos, disfagia e retardo no ganho de peso. ${ }^{16}$ Pode ocorrer retardo na marcha e ao adquirir movimentos finos das mãos em lactentes, geralmente transitórias. Displegia espástica pode ocorrer em até 20\% dos casos. ${ }^{4}$ Devem ser monitoradas durante o tratamento as funções hematológica, renal, tireoidiana, hepática, além de ser recomendado um acompanhamento neurológico durante e após o tratamento.

Anger et al sugeriram a retirada gradual da medicação após longo período de tratamento (6 a 24 meses) para evitar efeito rebote observado em sua experiência. ${ }^{17}$

Apesar de pouco esclarecimento a respeito de suas ações, a terapia com interferon alfa tem sido uma importante opção de tratamento para crianças com hemangiomas com- plicados, resistentes ao uso de corticoesteróides e com potencial de deformidades cosméticas ou de ameaça à vida.

\section{Abstract}

The aim of this study is to describe the treatment using interferonalpha of giant hemangiomas in children. The authors report two cases of children presenting with giant hemangiomas treated using interferon-alpha and analyze the results. IBS, 3 years-old, has been followed up in Famema Hemathology Service since she was 1 yearold with a tumor on the face and persistent bleeding. After clinical and radiologic evaluations and suggested the diagnosis of giant hemangioma, she started treatment with interferon-alpha. A great clinical improvement was observed a reducing of the number of episodes of bleedings and a decrease in of the tumor size. CNP, 12 years-old, came to this service in the last year presenting with a small painful tumor on the left knee. She had already tried a treatment with Prednisone with no improvement. Treatment with interferon-alpha was initiated with a significant decrease in its size. The use of interferon-alpha should be considered in the treatment of giant hemangioma due to its favorable results related to a reduction in the tumor size and the episodes of bleeding. Rev. bras. hematol. hemoter. 2007;29(4):406-411.

Key words: Hemangioma; alpha-interferon; bleeding; child.

\section{Referências Bibliográficas}

1. Finn MC, Glowacki J, Mulliken JB. Congenital vascular lesions: clinical application of a new classification. J Pediatr Surg 1983; 18:894.

2. Gontijo B, Silva CMR, Pereira LB. Hemangioma da infância. An Bras Dermatol. 2003;78(6):651-73.

3. Mulliken JB, Glowacki J. Hemangiomas and vascular malformations in infants and children: a classification based on endothelial characteristics. Plat Reconstr Surg. 1982;69(3):412-20.

4. Chiller KG, Passaro D, Frieden IJ. Hemangiomas of infancy: clinical characteristics, morphologic subtypes and their relationship to race, ethnicity and sex. Arch Dermatol. 2002;138:1567-76.

5. Damas MB, Menéndez SJJ, Abelairas GJM et al. Interferon alfa and infantile orbital hemangioma. Arch Soc Esp Oftalmol. 2003; 78(11):623-9.

6. Silva CMR, Viana MB, Gontijo B et al. Hemangioma gigante tratado com interferon alfa-2a. J Ped. 1997;73(4):277-80.

7. Christison-Lagay ER, Burrows PE, Alomari A et al. Hepatic hemangiomas: subtype classification and development of a clinical practice algorithm and registry. J Pediatr Surg. 2007;42:62-8.

8. Campos HGA, Curado JH. Angiodisplasias. In: Pitta GBB; Castro AA, Burihan E. Angiologia e Cirurgia Vascular: guia ilustrado. Maceió: Uncisa/Ecmal \& Lava, 2003.

9. Costa CML, Rodrigues KES, Campos HGA et al. Hemangioma hepático. J Ped. 2000;76(1):87-90.

10. Drolet BA, Esterly NB, Frieden IJ. Primary care: hemangiomas in children. N Engl J Med. 1999;341(3):173-81.

11. Haggstrom AN, Drolet BA, Baselga E et al. Prospective Study of Infantile Hemangiomas: Clinical characteristics predicting complications and treatment. Pediatrics. 2006;118(3):882-7.

12. Greinwald JH, Burke D, Bonthius D et al. An update on the treatment of hemangiomas in children with interferon alfa-2a. Arch Otolaryngol Head Neck Surg. 1999;125(1):21-7. 
13. Chang E, Boyd A, Nelson CC et al. Successful treatment of infantile hemangiomas with interferon alfa-2b (Case Reports). J Pediatr Hematol Oncol. 1997;19(3):237-44.

14. Hagberg H, Lamberg K, Astrom G. a-2b Interferon and oral clodronate for Gorham's disease. Lancet. 1997;350:1822-3.

15. Takahashi A, Ogawa C, Takashi K et al. Remission induced by interferon alfa in patient with massive osteolysis and extension of lymph-hemangiomatosis: a severe case of Gorham-Stout syndrome. J Pediatr Surg. 2005;40:E47-E50.

16. Szymik-kantorowicz S, Kobylarz K, Krysta M et al. Interferon-a in the treatment of high-risk haemangiomas in infants. Eur J Paediatr Surg. 2005;15:11-6.

17. Anger J, Carneiro RG, Pinus $\mathrm{J}$ et al. The rebound effect in the treatment of complex hemangioma with interferon alpha 2A. Rev Paul Med. 1998;116(5):1826-8.

Avaliação: Editor e dois revisores externos

Conflito de interesse: não declarado.

Recebido: 02/05/2006

Aceito após modificações: 02/01/2007 